\title{
Ultrasonic propagation in amorphous PdSiCu at low temperatures
}

\author{
P. Doussineau \\ Laboratoire d'Ultrasons $\left({ }^{*}\right)$, Université Pierre-et-Marie-Curie, Tour 13, 4, place Jussieu, 75230 Paris Cedex 05, France \\ (Reçu le 3 novembre 1980, accepté le 6 janvier 1981)
}

\begin{abstract}
Résumé. - L'atténuation et la variation de la vitesse de phase d'ondes ultrasonores ont été mesurées dans l'alliage amorphe $\mathrm{Pd}_{0,775} \mathrm{Si}_{0,165} \mathrm{Cu}_{0,06}$ en fonction de l'amplitude dans la gamme de fréquence 370 à $1080 \mathrm{MHz}$ et pour des températures aussi basses que $0,05 \mathrm{~K}$. L'atténuation dépendant de l'amplitude, attribuée à l'absorption résonnante par les systèmes à deux niveaux, varie comme le carré de la fréquence et l'inverse de la température, en accord avec le comportement prédit par la théorie des systèmes à deux niveaux. L'atténuation à forte amplitude varie linéairement avec la température et est indépendante de la fréquence aux plus basses températures. La vitesse augmente comme le logarithme de la température. Aucune variation en fonction de l'amplitude et de la fréquence de la pente de $\Delta v / v$ en fonction de Ln $T$ n'a été observée. Ainsi aucune confirmation du comportement observé à plus basse fréquence (10 à $90 \mathrm{MHz}$ ) par d'autres auteurs dans un alliage de même composition n'a pu être apportée.
\end{abstract}

\begin{abstract}
Attenuation and phase velocity change of ultrasonic waves have been measured in the amorphous metal $\mathrm{Pd}_{0.775} \mathrm{Si}_{0.165} \mathrm{Cu}_{0.06}$ as a function of the amplitude in the frequency range 370 to $1080 \mathrm{MHz}$ and for temperatures down to $0.05 \mathrm{~K}$. The amplitude dependent attenuation, attributed to the resonant absorption by the two-level systems (T.L.S.), varies as the square of the frequency and the inverse of the temperature, in agreement with the standard theory of T.L.S. The attenuation at high amplitude depends linearly on the temperature and is frequency independent at the lowest temperatures. The velocity increases logarithmically with the temperature. The slope of $\Delta v / v$ versus $\operatorname{Ln} T$ was found to be amplitude and frequency independent. Thus no confirmation of the behaviour observed at lower frequencies $(10$ to $90 \mathrm{MHz}$ ) by other workers in an alloy of the same composition could be provided.
\end{abstract}

1. Introduction. - During the past decade the existence of two-levels tunnelling systems (T.L.S.) has been experimentally demonstrated in amorphous insulators [1]. In the last three years the existence of T.L.S. has been also detected in amorphous metals by thermal conductivity experiments [2] and most significantly by ultrasonic phase velocity change [3] and saturation of the acoustic attenuation at low temperatures [4]. At this time it was believed that the low temperature properties of amorphous insulators and amorphous metals were similar even if one excepts some differences that are expected owing to the coupling between T.L.S. and conduction electrons in metals [5]. Recently some problems arose from the results of acoustic experiments at relatively low frequency (10 to $90 \mathrm{MHz}$ ). It was observed that in that frequency range and for temperatures between 0.3 and $1 \mathrm{~K}$ the amorphous alloy $\mathrm{Pd}_{0.775} \mathrm{Si}_{0.165} \mathrm{Cu}_{0.06}$ presents an attenuation at low acoustic power (the so-called resonant attenuation) varying linearly with

$\left({ }^{*}\right)$ Associated with the Centre National de la Recherche Scientifique. frequency, and temperature independent. Moreover the acoustic velocity although it presented the usual logarithmic temperature dependence, was found to be power dependent [6]. These results are in contradiction with the $\omega^{2} / T$ dependence and the independence of the velocity variation versus the acoustic power predicted by the current theory of T.L.S. and observed in amorphous insulators [7]. Up to date the experiments in amorphous metals at frequencies higher than $100 \mathrm{MHz}$ are restricted to two independent measurements at $720 \mathrm{MHz}$ [4] and $960 \mathrm{MHz}$ [5] respectively. Therefore there is a need for an extensive study of the saturation effects as a function of the temperature and the frequency in the $\mathrm{GHz}$ range.

In that context I have performed new acoustic propagation experiments in a $\mathrm{PdSiCu}$ alloy of the same composition in the frequency range from 370 to $1080 \mathrm{MHz}$ and at temperatures down to $0.05 \mathrm{~K}$ in order to determine whether the frequency, temperature and power dependences found at lower frequencies were also verified at these higher frequencies. My results show unambiguously that in the above frequency range and from $0.05 \mathrm{~K}$ to $0.4 \mathrm{~K}$, the resonant attenuation in amorphous $\mathrm{PdSiCu}$ has an $\omega^{2} / T$ 
dependence. Moreover, no power dependence has been observed in the logarithmic temperature dependence of the velocity.

2. Experiments. - The sample is a

$$
\mathrm{Pd}_{0.775} \mathrm{Si}_{0.165} \mathrm{Cu}_{0.06}
$$

alloy obtained by a rapid quenching from the liquid phase. It is derived from the same ingot as those used in previous experiments $[4,8,9]$. It has the shape of a cylindrical rod of diameter $2 \mathrm{~mm}$ and $3.74 \mathrm{~mm}$ long.

The acoustic attenuation was measured by using a standard pulse echo technique. The accuracy was about $0.2 \mathrm{~dB} . \mathrm{cm}^{-1}$. The relative velocity variation was measured by an interferometric technique with an accuracy of about $2 \times 10^{-6}$. All these experiments were restricted to transverse waves only because of the higher amplitude of the effects for that polarization.

2.1 Resonant attenuation. - Some typical curves of the ultrasonic attenuation as a function of the temperature are presented in figure 1 for two amplitudes of the ultrasonic pulse, one in the saturated or

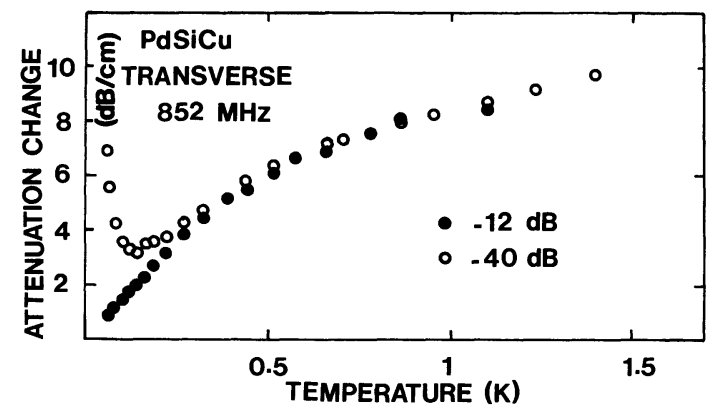

Fig. 1. - Temperature dependence of the attenuation of $852 \mathrm{MHz}$ transverse acoustic waves for two amplitudes in the amorphous metal $\mathrm{Pd}_{0.775} \mathrm{Si}_{0.165} \mathrm{Cu}_{0.06}$.

high power regime and the other in the non-saturated or low power regime. Clearly at low amplitude the attenuation at the lowest temperatures is higher than the attenuation at high amplitude. Moreover, below $0.15 \mathrm{~K}$ for the low amplitude curve the attenuation increases when the temperature decreases.

In order to study the resonant part in more detail, the attenuation was plotted as a function of the acoustic power at various temperatures. Some curves are presented in figure 2 for $852 \mathrm{MHz}$. The experimental curves are fitted with an expression of the form $\alpha_{\mathrm{R}}=\alpha_{0}\left(1+\frac{\Phi}{\Phi_{\mathrm{c}}}\right)^{-1 / 2}[1], \alpha_{0}$ and $\Phi_{\mathrm{c}}$ being adjustable parameters, $\Phi$ the acoustic flux in the sample, $\Phi_{\mathrm{c}}$ a critical flux. That expression results from the theory of T.L.S., which also gives [1]

$$
\alpha_{0}=\pi \frac{\bar{P} \gamma^{2}}{\rho v^{3}} \omega \tanh \frac{\hbar \omega}{2 k T},
$$

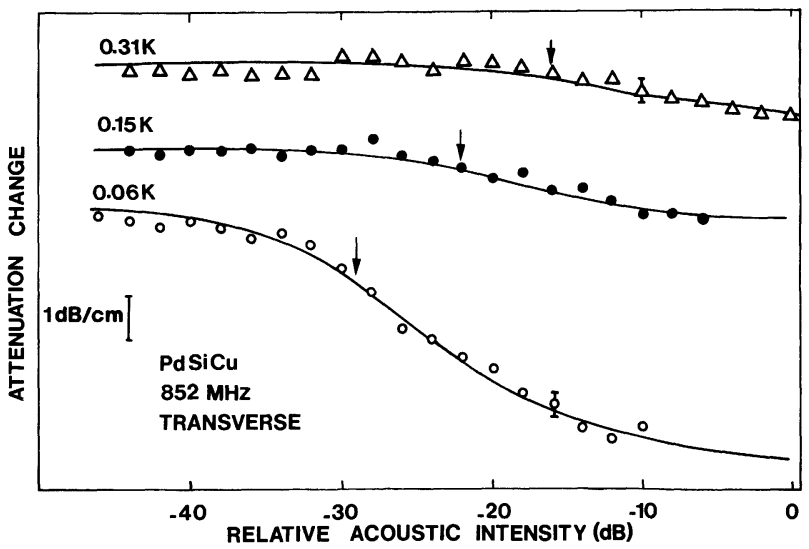

Fig. 2. - Amplitude dependence of the attenuation of $852 \mathrm{MHz}$ transverse acoustic waves in the amorphous metal

$$
\mathrm{Pd}_{0.775} \mathrm{Si}_{0.165} \mathrm{Cu}_{0.06}
$$

for various temperatures. The solid curves are calculated curves as indicated in the text. The arrows mark the values of $\Phi_{\mathrm{c}}$ obtained from the fitting. $0 \mathrm{~dB}$ corresponds roughly to an acoustical flux of $10^{-1} \mathrm{~W} \cdot \mathrm{cm}^{-2}$ in the sample.

where $\bar{P}$ and $\gamma$ are respectively the density of states of the T.L.S. and the coupling constant between the T.L.S. and the ultrasonic wave of frequency $\omega / 2 \pi$ propagating with the velocity $v, \rho$ is the mass density of the material. If $\hbar \omega \ll 2 k T, \alpha_{0}$ scales as $\omega^{2} / T$.

The values of $\alpha_{0}$ obtained from the fits of figure 2 and similar ones at various frequencies and temperatures are plotted in figure $3 a$ as a function of the frequency at two temperatures $(0.06 \mathrm{~K}$ and $0.1 \mathrm{~K})$. Clearly an $\omega^{2}$ dependence describes the results well. At $1 \mathrm{GHz}$ and $0.06 \mathrm{~K}$ the approximation

$$
\tanh \hbar \omega / 2 k T \simeq \hbar \omega / 2 k T
$$

is no longer valid but the difference is too small to be seen in this experiment. In figure $3 b$ the same quantity $\alpha_{0}$ is plotted as a function of the temperature at two frequencies. The data agree very well with a $T^{-1}$ dependence. Thus the $\omega^{2} T^{-1}$ dependence predicted by the standard two-level tunnelling theory
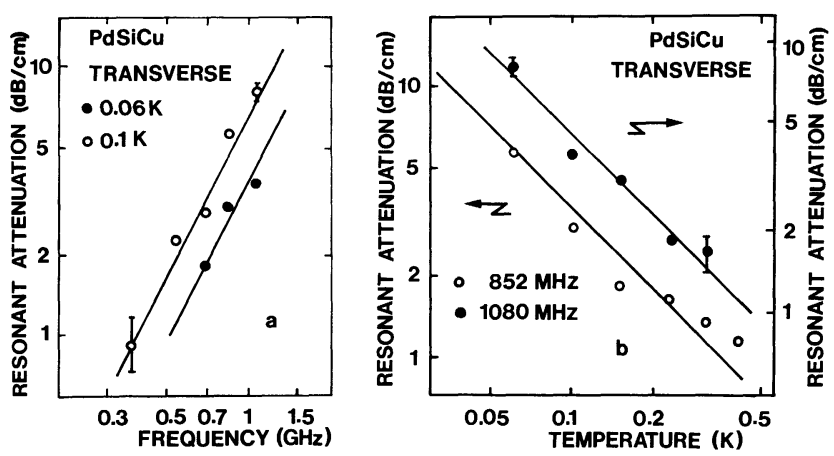

Fig. 3. - Ln-Ln plot of the resonant part of the acoustic attenuation in the amorphous metal $\mathrm{Pd}_{0.775} \mathrm{Si}_{0.165} \mathrm{Cu}_{0.06}$ as a function of the frequency $(a)$ at two temperatures and of the temperature $(b)$ at two frequencies. The solid lines correspond to a slope 2 in $(a)$ and -1 in $(b)$ respectively. 
is verified in the above experimental conditions $(370 \leqslant \omega / 2 \pi \leqslant 1080 \mathrm{MHz}, 0.05 \leqslant T \leqslant 0.4 \mathrm{~K})$ on the amorphous metal $\mathrm{PdSiCu}$.

2.2 Saturated attenuation. - The non-resonant (or relaxational) part of the attenuation [10], obtained with a high acoustic power, is shown in figure 4 at various frequencies. First, the attenuation increases linearly with the temperature with a slope which is independent of the frequency. When the

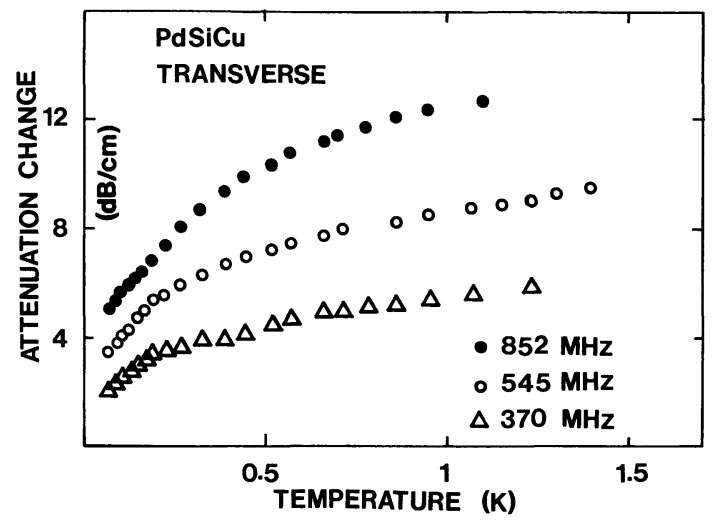

Fig. 4. - Attenuation change at high amplitude (relaxational attenuation) as a function of the temperature for three frequencies of the transverse acoustic waves propagating in an amorphous $\mathrm{Pd}_{0.775} \mathrm{Si}_{0.165} \mathrm{Cu}_{0.06}$ sample. The origin of the attenuation is arbitrary. The curves are arbitrarily shifted one from the other.

temperature increases further the attenuation becomes frequency dependent, varying roughly as $\omega T$, as has been shown previously [9]. Such a behaviour has already been observed in amorphous metal $\mathrm{Ni}_{0.78} \mathrm{P}_{0.22}$ [11]. It has been attributed to relaxation of the T.L.S. by the conduction electrons in the regime $\omega T_{1} \gg 1$, where $T_{1}$ is the relaxation time of the T.L.S. In that case the theory predicts [7, 11] :

$$
\alpha_{\mathrm{Rel}}=\pi^{3} \bar{P} \gamma^{2}(n K)^{2} k T / 24 \hbar \rho v^{3}
$$

where $n$ is the electronic density of states at the Fermi level and $K$ the coupling constant between T.L.S. and electrons. A full description of the attenuation curve requires a numerical calculation and probably must take into account the existence of a relaxation peak at higher temperature [12].

2.3 Phase Velocity VARiation. - The phase velocity variation was measured as a function of the temperature at various amplitudes of the acoustic wave. The results are shown in figure 5 at $852 \mathrm{MHz}$. Within the experimental accuracy the variation is amplitude independent. The data below $0.15 \mathrm{~K}$ were discarded because at high amplitude there are some heating effects on the sample.

The temperature variation is found to increase logarithmically, as is usually observed in amorphous materials $[1,3,8]$. In another experiment at higher temperatures a velocity maximum near $2.5 \mathrm{~K}$ is observed, followed by a decrease which cannot be

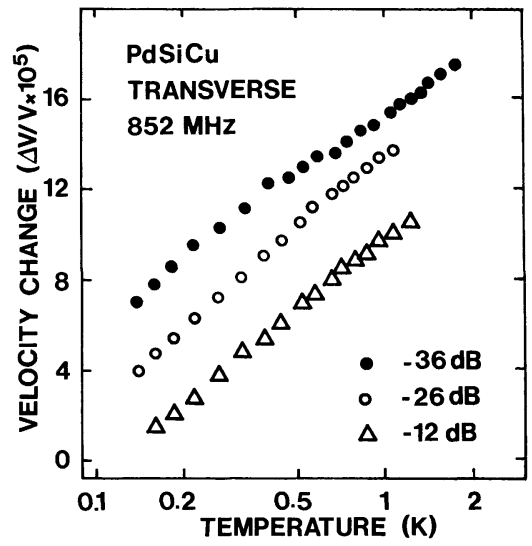

Fig. 5. - Temperature dependence of the velocity change of $852 \mathrm{MHz}$ transverse acoustic waves in the amorphous metal $\mathrm{Pd}_{0.775} \mathrm{Si}_{0.165} \mathrm{Cu}_{0.06}$ for three different amplitudes. The curves are arbitrarily shifted one from the another.

described by a linear temperature variation in the temperature range 5 to $30 \mathrm{~K}$. Moreover, no frequency dependence of the logarithmic part of the velocity was observed in the frequency range studied $(370 \mathrm{MHz}$ to $1080 \mathrm{MHz}$ ).

3. Discussion. - The experimental results reported above present clearly the following features :

- a saturable part of the ultrasonic attenuation which varies quadratically with the frequency. and as the inverse of the temperature;

- a non-saturable (power independent) part of the attenuation which at the lowest temperatures increases linearly with $T$ and is frequency independent ;

- a logarithmic increase of the phase velocity which is non-saturable and frequency independent.

All these features are in full agreement with the standard theory of two-level tunnelling systems developed for the description of the low temperature properties of amorphous materials [1, 13].

Unfortunately with the present experimental set-up, it was not possible to detect any saturation effects at temperatures higher than $0.4 \mathrm{~K}$ and at frequencies lower than $370 \mathrm{MHz}$. Thus the present frequency and temperature ranges do not overlap those studied by Araki et al. [6].

In their discussion, Araki et al. [6] argued from the temperature independence of $\alpha_{0}$ below about $1 \mathrm{~K}$, for extrapolating the result of Doussineau et al. (at $720 \mathrm{MHz}$ ) [4] from $0.06 \mathrm{~K}$ up to $0.3 \mathrm{~K}$. Evidently the results presented here argue against such an extrapolation.

From the experimental curves some parameters can be deduced. For the product $\bar{P} \gamma^{2}$ I find

$$
1.2 \times 10^{7} \mathrm{erg} . \mathrm{cm}^{-3}
$$

from the frequency dependence of $\alpha_{0}$,

$$
1.5 \times 10^{7} \mathrm{erg} . \mathrm{cm}^{-3}
$$


from the temperature dependence of $x_{0}$ and

$$
1.4 \times 10^{7} \mathrm{erg} . \mathrm{cm}^{-3}
$$

from the logarithmic increase of the velocity. These values are of the same order as those already published $[4-6,8,14]$. It must be noted that the values of $\bar{P} \gamma^{2}$ given here are roughly $30 \%$ lower than the value deduced from an earlier saturation experiment at $720 \mathrm{MHz}$ and $0.06 \mathrm{~K}$ [4] on a sample from the same ingot. It is difficult to explain this. Perhaps it is due to an annealing of the sample, although it was kept at room temperature between the two sets of experiments. The values of the critical acoustic flux $\Phi_{c}$ are in agreement with previously published values $[4,5]$. For example at $0.06 \mathrm{~K}$ and $852 \mathrm{MHz}$, $\Phi_{\mathrm{c}} \simeq 10^{-4} \mathrm{~W} . \mathrm{cm}^{-2}[15]$. At $852 \mathrm{MHz} \Phi_{\mathrm{c}}$ was found to follow roughly a quadratic variation with the temperature in the range $0.06 \mathrm{~K}$ to $0.4 \mathrm{~K}$. Araki et al. [6] found the same variation in the 10 to $100 \mathrm{MHz}$ range from $0.3 \mathrm{~K}$ to $2 \mathrm{~K}$.

From the linear part of the temperature dependence of the relaxation attenuation a value for the product of the electronic density of states at the Fermi level by the coupling constant between electrons and T.L.S. is deduced. I find $(n K) \simeq 0.3$, a value near previous independent determinations in $\mathrm{PdSiCu}$ [5] or NiP [11].

4. Conclusion. - In the temperature $(0.05 \leqslant T \leqslant$ $0.4 \mathrm{~K})$ and frequency $(370 \leqslant \omega / 2 \pi \leqslant 1080 \mathrm{MHz})$ ranges studied the amorphous metal $\mathrm{PdSiCu}$ follows the current two-level tunnelling theory very well. No confirmation of the behaviour observed at lower frequencies (10 to $90 \mathrm{MHz}$ ) by Araki et al. [6] on an alloy of the same composition could be provided.

\section{References}

[1] Hunklinger, S. and Arnold, W., Physical Acoustics, edited by W. P. Mason and R. N. Thurston (Academic Press, New York) 1976, volume 12, p. 155.

[2] Matey, J. R. and Anderson, A. C., J. Non-Cryst. Solids 23 (1977) 129; Phys. Rev. B 16 (1977) 3406.

[3] Bellessa, G., Doussineau, P. and Levelut, A., J. Physique Lett. 38 (1977) L-65.

[4] Doussineau, P., Legros, P., Levelut, A. and Robin, A., J. Physique Lett. 39 (1978) L-285.

[5] Golding, B., Graebner, J. E., Kane, A. B. and Black, J. L., Phys. Rev. Lett. 41 (1978) 1487.

[6] Araki, H., Park, G., Hikata, A. and Elbaum, C., Solid State Commun. 32 (1979) 625; Phys. Rev. B 21 (1980) 4470.

[7] BLACK, J. L., in Metallic Glasses, edited by H. J. Guntherodt (Springer Verlag, New York), to be published.

[8] Bellessa, G. and Bethoux, O., Phys. Lett. 62A (1977) 125.
[9] Doussineau, P., Levelut, A., Bellessa, G. and Bethoux, O., J. Physique Lett. 38 (1977) L-483.

[10] JäCKLE, J., Z. Phys. 257 (1972) 212.

[11] Doussineau, P. and Robin, A., Proc. 3rd Internat. Conf. on Phonon scattering in condensed matter, edited by $\mathrm{H}$. J. Maris (Plenum Publ. Co, New York) 1980, p. 65.

[12] Dutoit, M., Phys. Lett. 50A (1974) 221.

[13] Phillips, W. A., J. Low Temp. Phys. 7 (1972) 351 ;

Anderson, P. W., Halperin, B. I. and Varma, C. M., Philos. Mag. 25 (1972) 1.

[14] Matey, J. R. and Anderson, A. C., Phys. Rev. B 17 (1978) 5029.

[15] As usual in ultrasonic experiments the absolute value of the acoustic flux in the sample is difficult to know with high accuracy. It is only known within a factor about 10 . However the relative variations of the acoustic flux are well determined. The accuracy is about $0.2 \mathrm{~dB}$. 